\title{
La mediación cultural digital en el ámbito educativo: Los programas educativos del MUCEN como un recurso pedagógico en tiempos de pandemia
}

\section{Jorge Manuel Kishimoto Pinillos ${ }^{\mathrm{a}}$ y Claudia Stephanie Sánchez Salazar}

${ }^{\mathrm{a} B a n c o}$ Central de Reserva del Perú, jorge.kishimoto@bcrp.gob.pe ${ }^{\mathrm{b}}$ Banco Central de Reserva del Perú, claudia.sanchez@bcrp.gob.pe

\begin{abstract}
Resumen
Los programas educativos del Museo Central respondieron al desafio de la interacción digital y a la complejidad de afrontar la educación en remoto producto de la pandemia del COVID-19. Estos están compuestos por recursos educativos digitales, tales como videos interactivos, guías, fichas y cartillas metodológicas dirigidas a docentes y estudiantes de nivel primario y secundario a nivel nacional. Los recursos fueron elaborados utilizando las metodologías de la pedagogía en museos y las herramientas de la mediación cultural para llevar la experiencia del museo al aula.
\end{abstract}

La primera fase de evaluación de los programas "Cosmovisiones" y "Nuestra vestimenta" evidencia una buena recepción de los recursos desarrollados, así como un panorama favorable para continuar con el diseño y ejecución de estos. Los docentes deben ser los principales aliados en la implementación de los programas, ya que sin ellos asumiendo el rol de mediadores culturales no se lograrían los objetivos de aprendizaje esperados. Por ello, el MUCEN apuntará a fortalecer las capacidades y los vínculos con los docentes aliados a través de espacios de formación y creación colectiva.

Palabras clave: Programas educativos, recursos digitales, metodologías pedagógicas, docente aliado 


\begin{abstract}
The Museo Central's educational programs responded to the challenge of digital interaction and the complexity of facing remote education due to the COVID-19 pandemic. These are made up of digital educational resources, such as interactive videos, guides, cards, and methodological booklets aimed at teachers and students at the primary and secondary level nationwide. The resources were prepared using museum pedagogy and cultural mediation tools to bring the museum experience to the classroom.

The first phase of evaluation of the programs called "Cosmovisiones" and "Nuestra Vestimenta" shows a good reception of the developed resources as well as a favorable picture to continue with their design and execution. Teachers shall be the main allies in implementing the programs since without their role as cultural facilitators, the expected learning objectives could not be achieved. For this reason, the MUCEN will aim to strengthen capacities and ties with allied teachers through training and collective creation spaces.
\end{abstract}

Keywords: educational programs, digital resources, museum pedagogy, allied teachers 


\section{Introducción}

El Museo Central (MUCEN) fue creado en 1982 por el Banco Central de Reserva del Perú como parte de su política cultural centrada en brindar libre acceso a la cultura e incentivar la producción artística nacional. El MUCEN ha desarrollado una larga y activa trayectoria que lo constituye como un espacio cultural sólido, que contribuye a dinamizar el sector cultural peruano. Hoy en día, el Museo Central es una red de museos con presencia en diferentes departamentos del Perú como Lima, Arequipa, Piura y Trujillo, en el que el diálogo es usado como herramienta para generar comunidad; un espacio para interactuar con el arte y con otros visitantes.

El MUCEN desarrolla una labor de gestión, conservación e investigación de colecciones conformada por la colección numismática, la colección de arte colonial, republicano y contemporáneo y la colección de arte precolombino formada por 28 mil piezas a las que se puede acceder desde nuestro Sistema de Colecciones ${ }^{1}$ albergado en la web.

Respondiendo al contexto del S.XXI y como resultado de un proceso de repensar el rol del museo en relación a la comunidad que sirve, el MUCEN replanteó su misión -en línea con sus políticas culturales- para enfatizar la importancia de "contribuir al fortalecimiento de la ciudadanía a través del conocimiento y la valoración de la diversidad cultural peruana de todos los tiempos". Para ello, el enfoque pedagógico es la principal estrategia implementada de forma transversal a todos los programas del museo.

La pandemia del COVID-19 generó un cambio radical en la forma en la que el MUCEN interactuaba con sus públicos. Por ello, buscó fomentar la creación de nuevos espacios de interacción y encuentro desde lo digital para continuar con su misión. Por un lado, el museo se vio distanciado de su público principal, los grupos escolares que habitualmente nos visitaban y, por otro lado, los docentes se enfrentaron al reto de la educación remota y digital en tiempos de incertidumbre.

En este contexto, el MUCEN realizó un diagnóstico a través de una encuesta con el objetivo fortalecer sus vínculos con la comunidad docente y conocer a través de qué recursos digitales el museo podría contribuir mejor con su labor. En esta participaron 828 docentes de primaria y secundaria, tanto de escuela pública como privada, a nivel nacional, que proporcionaron información valiosa para explorar nuevas rutas de colaboración entre museos y escuelas.

Los resultados obtenidos en la encuesta fueron utilizados por el área educativa como base para desarrollar diversos tipos de recursos educativos en formato digital, tales como series de podcast vinculadas a las historias detrás de las piezas del museo ${ }^{2}$, recorridos virtuales en $360^{\circ 3}$ y la digitalización de programas educativos integrales. Este trabajo nos ha permitido continuar cumpliendo con la misión del MUCEN aún de forma remota.

El presente artículo explorará los casos de digitalización de los programas educativos del Museo Central que respondieron al desafío de la interacción digital. Esta propuesta educativa integral está vinculada al Currículo Nacional de la Educación Básica y consta de una serie de

\footnotetext{
${ }^{1}$ Sistema de Colecciones del MUCEN: https://mucen.bcrp.gob.pe/museo/

${ }^{2}$ Ver repositorio de Series podcast: https://www.bcrp.gob.pe/museocentral/podcast-mucen.html

${ }^{3}$ Recorridos virtuales: https://www.bcrp.gob.pe/museocentral/exposiciones/exposiciones-permanentes.html
} 
recursos audiovisuales que buscan incentivar el diálogo, la creación y los nuevos aprendizajes, así como herramientas y materiales pedagógicos (guía docente y fichas educativas) diseñados para que los docentes asuman el rol de mediadores culturales en clase.

Los programas educativos en versión digital "Cosmovisiones" y "Nuestra vestimenta" invitan a recorrer el museo y explorar obras de arte peruano de la colección del MUCEN a través de una serie de videos. Ambas propuestas fomentan el respeto y la valoración a la diversidad cultural con el objetivo de construir una ciudadanía intercultural.

Finalmente, se comentará a partir de la experiencia en el desarrollo y aplicación de estos programas, las oportunidades y los aspectos claves en el proceso de la mediación digital, así como en la importancia de desarrollar acciones colectivas que fortalezcan los vínculos entre instituciones culturales y educativas. Desde el MUCEN seguiremos explorando nuevas rutas para transformarnos en verdaderos agentes sociales a través de proyectos que contribuyan a la formación de ciudadanas y ciudadanos más empáticos, solidarios y comprometidos con la sociedad.

\section{Desarrollo de la innovación}

\subsection{Diagnóstico}

Ante el difícil contexto de distanciamiento físico, se decidió realizar a manera de diagnóstico la "Encuesta para docentes: Recursos educativos del Museo Central"4 del 23 de abril al 3 de mayo de 2020. Esta tenía por objetivo identificar las principales necesidades de recursos educativos de los docentes en el marco de la educación remota. Como resultado, se contó con la participación de 828 docentes de nivel primario y secundario a nivel nacional.

Esta encuesta se estructuró en tres grandes partes. La primera corresponde al perfil de los docentes encuestados que nos permitió conocer mejor sus edades promedio, el departamento donde residen, los niveles en los que enseñan, el área curricular y el tipo de institución educativa donde laboran. En la segunda parte se les presentó una serie de recursos educativos digitales con el objetivo de que nos indicaran su grado de interés, así como el tiempo que le dedicarían a su uso en clase. Además, en esta sección los invitamos a compartir con nosotros sus temas de interés y su manera de concebir el museo respecto a su labor pedagógica. Finalmente, buscamos conocer la frecuencia con la cual utilizan la metodología de aprendizaje basada en proyectos y algunos ejemplos de estos que hayan aplicado en clase.

Como resultado del análisis de los datos obtenidos, pudimos observar que existía una gran demanda entre los docentes encuestados por todos los materiales educativos propuestos. Así mismo, el recurso preferido fue el video didáctico para utilizar en clase que obtuvo un $92,5 \%$

\footnotetext{
${ }^{4}$ Informe de Resultados "Encuesta para docentes: Recursos educativos del Museo Central" https://www.bcrp.gob.pe/docs/Proyeccion-Institucional/Museo/encuesta-docente-2020.pdf
} 
de interés como se observa en el Gráfico 1. Esto nos llevó a reflexionar sobre la importancia de diseñar programas educativos integrales que incluyan múltiples materiales orientados tanto al aprendizaje de los estudiantes como al acompañamiento y formación de los docentes.

Gráfico 1. ¿Qué recursos digitales te interesaría que el MUCEN desarrolle para contribuir con tu labor docente?

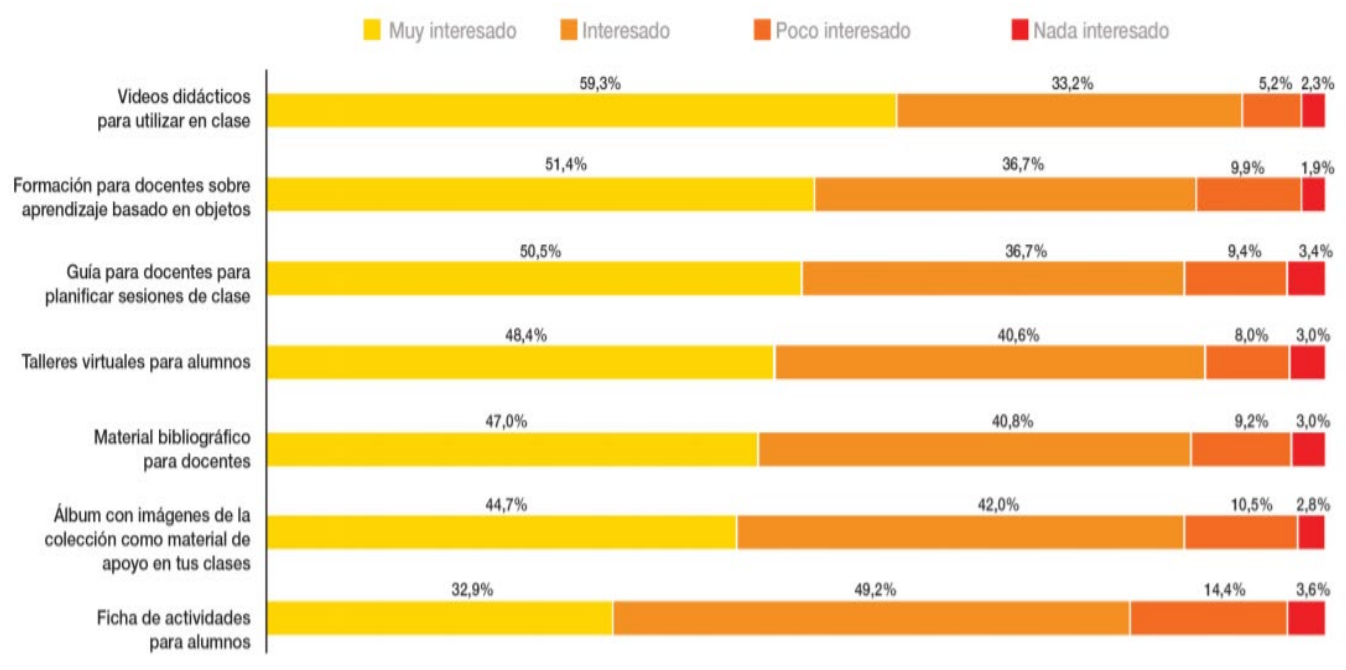

Otro de los resultados fundamentales obtenidos estuvo relacionado al tiempo promedio que los docentes dedicarían a realizar programas educativos propuestos por el museo. Este oscila entre los 15-30 minutos de su sesión de clase aproximadamente. Sin embargo, en programas como la maleta didáctica, que contienen diversos recursos y actividades para incentivar el aprendizaje de los estudiantes, y los talleres artísticos virtuales para fomentar su creatividad están dispuestos a emplear un tiempo mayor o incluso más de una sesión de clase. Estos resultados nos llevaron a estructurar los programas educativos de manera que los docentes puedan utilizarlos en una o múltiples sesiones de clase sin que esto afecte la experiencia de aprendizaje. 
Gráfico 2. ¿Cuánto tiempo de tu clase le dedicarías a los siguientes programas?

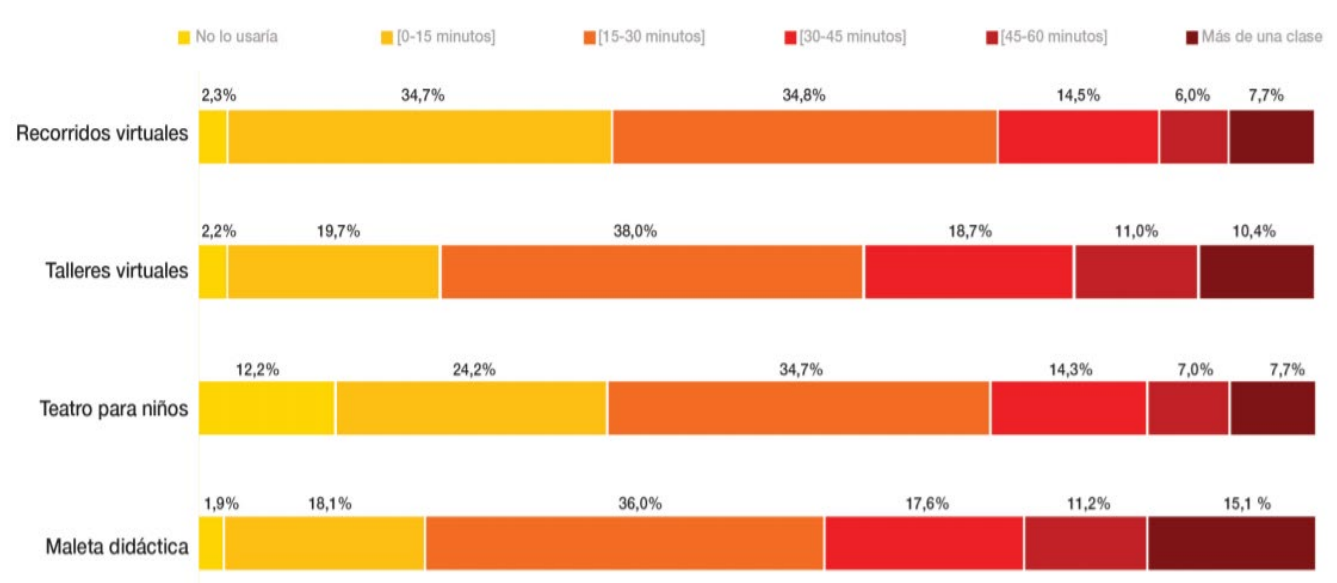

Es importante resaltar que el mayor número de docentes encuestados pertenecía al área curricular de Ciencias Sociales. Sin embargo, contamos con una alta participación de docentes de múltiples áreas que tradicionalmente no se relacionan con museos de arte tales como Matemática, Ciencia y Tecnología y Comunicación. Esto nos permitió explorar la posibilidad de plantear los programas educativos en versión digital desde una perspectiva multidisciplinaria, los cuales cuenten con actividades y proyectos de aprendizaje que inviten a colaborar entre las distintas asignaturas.

Finalmente, es a partir de este diagnóstico que desde el área de educación del MUCEN identificamos las principales rutas que debíamos seguir en la construcción de los programas educativos en versión digital. Estos debían ser programas integrales, que incluyan un paquete de recursos educativos diversos tanto para estudiantes como para docentes y que sean flexibles para el uso en las sesiones de clases dependiendo del tiempo con el que cuenta cada docente. Así mismo, estos debían generar conexiones interdisciplinarias entre las áreas de Arte y Cultura y Ciencias Sociales con el resto de asignaturas de manera innovadora y positiva.

\subsection{Antecedentes}

El Museo Central ofrecía seis programas educativos para escolares, diseñados por niveles específicos, que consistían en recorridos temáticos en los que se exploraba hasta 5 piezas de las colecciones de arte del museo a través del diálogo y la interacción. Tan solo en el año 2019, el MUCEN recibió un promedio de 28000 estudiantes de escuelas públicas y privadas 
que realizaron estos recorridos llamados "ConversArte"5 que abordan variados temas de interés ciudadano y educativo.

Estos recorridos temáticos se realizaban únicamente de manera presencial con los grupos escolares que visitaban el museo. Por ello, estaban centrados en brindar una experiencia durante el tiempo que el grupo permanecía en las salas de exposición. Sin embargo, tanto alumnos como docentes, por lo general, desconocían la temática del recorrido y no se contaban con recursos educativos para profundizar la experiencia antes o después de la visita

A partir de la revisión del diagnóstico y de los programas educativos con los que contaba el MUCEN de manera presencial, el equipo de educación asumió el reto de empezar a digitalizar estos dotándolos de nuevos contenidos, actividades, proyectos $\mathrm{y}$, sobre todo, desarrollando una gran cantidad de material educativo compatible con el medio digital tales como videos, guías, cartillas y fichas con el objetivo de facilitar el aprendizaje escolar.

Si bien la relación entre museos y escuelas data de hace muchos años, las acciones educativas han sido en muchos casos de carácter contemplativo y en mucha menor medida propician situaciones de participación y comunicación (Asensio y Pol, 2008). Por ello, la elaboración de estos programas educativos tiene por objetivo que los docentes se conviertan en aliados y asuman el rol de mediadores culturales, fomentando el diálogo y la participación a través de actividades y proyectos que generen aprendizajes significativos en sus alumnos.

\subsection{Programas educativos en versión digital}

Una de las rutas identificadas a partir de la encuesta a docentes fue la necesidad de diseñar programas integrales, es decir que incluyan recursos digitales no solo para estudiantes sino también para docentes, con el fin de servir como material de guía y de formación. Por ello, durante el año 2020 se desarrollaron los programas educativos en versión digital "Nuestra vestimenta" y "Cosmovisiones"6, tomando como base los recorridos temáticos realizados de manera presencial antes de la pandemia.

En vista de que los educadores y mediadores del MUCEN se encontraban alejados de las salas de exhibición, se buscó trasladar la experiencia de recorrer el museo a las aulas virtuales mediante videos interactivos. Para ello, los guiones fueron diseñados haciendo uso de las metodologías que se impartían en sala, como la mediación cultural, el aprendizaje basado en objetos y las estrategias de formulación de preguntas.

Asimismo, se buscó mantener los vínculos que tiene el museo con las distintas comunidades de visitantes y seguir democratizando el acceso a los programas educativos a través de la

\footnotetext{
${ }^{5}$ Los programas educativos "ConversArte" están alineados al Currículo Nacional del Ministerio de Educación y cuentan con objetivos específicos. https://www.bcrp.gob.pe/docs/Proyeccion-Institucional/Museo/memoriamucen-2018.pdf

${ }^{6}$ Programas educativos digitales MUCEN: https://www.bcrp.gob.pe/museocentral/aprende.html
} 
inclusión de un intérprete de Lengua de Señas Peruana en los materiales audiovisuales desarrollados ${ }^{7}$.

Cada programa educativo en versión digital consta de una serie de cuatro videos interactivos y fichas de trabajo para los estudiantes según su edad y nivel educativo que permitan reforzar los contenidos del programa, así como fomentar la creación y la investigación.

Para el Museo Central la mediación cultural es una metodología esencial en el ámbito educativo, ya que fomenta la interacción y el diálogo entre los visitantes y las colecciones del museo. Además, promueve las experiencias sensoriales, el pensamiento crítico y enriquece la exploración visual del entorno que nos rodea. Es por ello que, para asegurar el éxito en la aplicación de los programas, el museo ha desarrollado recursos complementarios para que los docentes asuman el rol de mediadores culturales en sus aulas. Este es el caso de la guía para docentes y de las cartillas metodológicas que brindan herramientas para estructurar sesiones de clase y proyectos de aprendizaje.

\subsection{Metodologías}

\subsubsection{Mediadores en las aulas}

Los programas educativos se encuentran vinculados con el Currículo Nacional ${ }^{8}$ y se orientan a una aplicación interdisciplinaria en la programación curricular. Así, se abordan temas sociales, culturales e históricos que promueven la colaboración entre docentes de diversas asignaturas y permiten a los alumnos reforzar competencias y capacidades propias de su nivel educativo.

Un docente de Ciencia y Tecnología, por ejemplo, podrá explorar en clase la pintura "Cosmovisión Bora" (1998) del artista Víctor Churay e inspirar a sus alumnos a desarrollar la actividad sobre flora y fauna de la Amazonía (Fig.1), propuesta en la "Guía para docentes". Por otro lado, un profesor de Comunicaciones podría narrar los mitos y leyendas representados en dicha obra de arte. Así, a través de los recursos propuestos en los programas educativos resaltamos el rol de las colecciones del museo como fuentes valiosas de información que constituyen un testimonio directo del pasado y del presente, donde una sola pieza puede ser analizada desde nuevos puntos de vista y diversas disciplinas.

\footnotetext{
${ }^{7}$ Recursos digitales desarrollados por el MUCEN https://www.bcrp.gob.pe/museocentral/recursos-digitalesmucen.html

${ }^{8}$ Documento marco de la política educativa de la educación básica desarrollada por el Ministerio de Educación del Perú.
} 

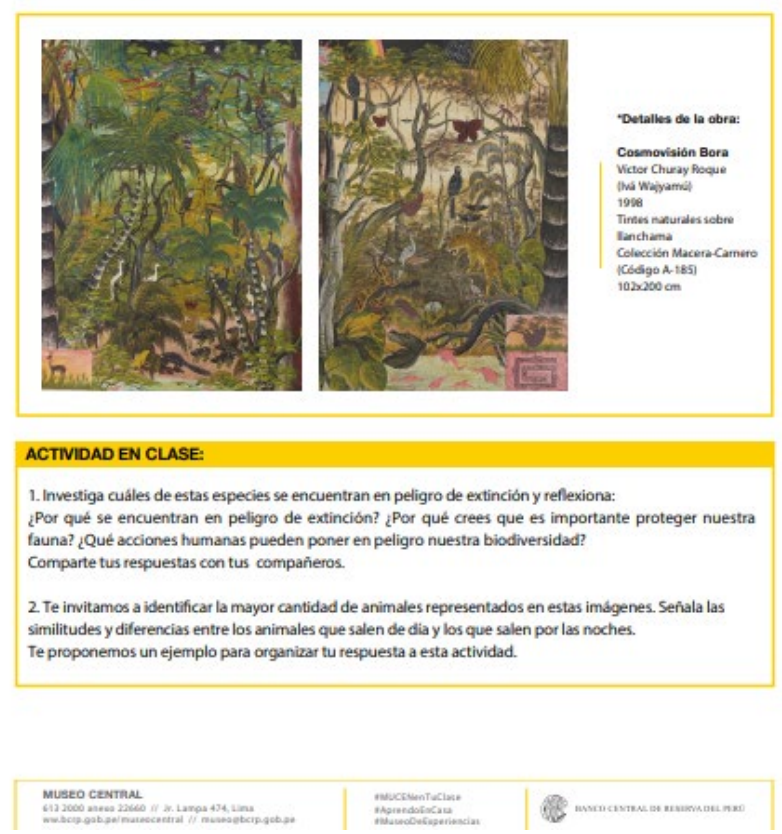

Fuente: Web MUCEN

\section{Fig. 1: Ficha educativa "Descubriendo la fauna de la Amazonía"}

Como resalta Alderoqui en el libro Museos y escuelas: socios para educar, el museo no es una escuela, y tiene dinámicas y estrategias distintas de generar aprendizajes desde "lo romántico, emotivo, activo, interactivo y reflexivo." (1996, p. 37). Es por esta razón, que planteamos los programas educativos digitales con recursos para antes, durante y después del recorrido temático, de manera que los alumnos y alumnas tengan la oportunidad de reflexionar, crear, interactuar y afianzar conocimientos y experiencias.

- "Prepárate para el recorrido" tiene el objetivo de invitar a los alumnos a la reflexión en torno a la temática del programa mediante un video introductorio y una actividad para motivar a la clase.

- "Desarrolla el programa" propone explorar las obras a través de tres videos interactivos que permitan generar diálogos en el aula y realizar microactividades complementarias. 
- "Amplía la experiencia" contiene sugerencias de proyectos de aprendizaje interdisciplinarios y actividades para utilizar en futuras sesiones.

La metodología de trabajo por etapas se traslada también al ámbito de evaluación de los programas, ya que antes de la presentación del recorrido "Nuestra Vestimenta", tuvimos una experiencia de evaluación con docentes de la Institución Educativa 1038 María Parado de Bellido, a través de una sesión con 8 docentes de primaria, en la que se evaluaron:

a. La coherencia temática y curricular del Programa educativo "Nuestra Vestimenta" para el nivel primaria alta.

b. La percepción de la innovación educativa.

c. Nuevas rutas de exploración desde el uso de los recursos del programa y,

d. La pertinencia del empleo de materiales educativos desarrollados en el ámbito escolar.

Los resultados obtenidos nos permitieron identificar que nuestros contenidos generaron curiosidad e interés en docentes y estudiantes por investigar temas complementarios. Así mismo, gracias a los comentarios de los docentes pudimos afinar el material desarrollado y conocer más sobre la necesidad de contar con recursos educativos de calidad, además de espacios de formación y acompañamiento en la aplicación de programas desarrollados por museos.

\subsubsection{El museo constructivista}

Haciendo eco a las teorías constructivistas de la educación, los museos han ido cambiando el paradigma de una educación basada en la contemplación pasiva donde la institución proveía de conocimiento, a una en la que el visitante es fuente y generador de saberes, cumpliendo un rol protagónico.

George E. Hein (1998) plantea en su libro Learning in the museum, el término "museo constructivista" para identificar a aquellas instituciones que ven al estudiante o visitante como centro del proceso de aprendizaje, tomando en cuenta las distintas formas que existen para aprender. Desde el MUCEN tomamos como referencia también la teoría de las inteligencias múltiples planteadas por Gardner (1985) para promover aprendizajes desde la diversidad de habilidades y capacidades de las personas, pero también desde las diversas motivaciones y necesidades de nuestros públicos.

Así, por ejemplo, en el programa educativo en versión digital "Nuestra vestimenta" invitamos a los docentes a desarrollar la inteligencia musical de sus alumnos, a través de una actividad para reflexionar en torno a los significados y usos de los objetos de metal en la vestimenta tradicional peruana. La actividad propone interpretar los sonidos que generan los cascabeles en la vestimenta utilizada en la danza afroperuana "Hatajo de Negritos". Esto permite que los estudiantes puedan identificar que a través de la música, instrumentos y sonidos en la vestimenta se pueden transmitir mensajes y emociones. 
Asimismo, nuestras propuestas de proyectos de aprendizaje, incentivan tanto la inteligencia interpersonal como la intrapersonal, ya que promueven la participación de los estudiantes durante todo el proceso y propone altas cuotas de comunicación e interacción, no solo entre estudiantes sino también con otros agentes sociales como las familias. Desde la práctica educativa del MUCEN, las teorías de las inteligencias múltiples son tomadas como base también para la aplicación de metodologías pedagógicas basadas en experiencias sensoriales y los diálogos sostenidos a través de preguntas.

\subsubsection{El poder de los objetos}

Los objetos o artefactos son el principal recurso de los museos y la base de la labor educativa no solo por la información que es posible obtener del objeto, sino porque estos nos permiten conocer las historias de sus creadores. Estos también nos brindan información de una sociedad en específico y de una realidad pasada. Los objetos pueden ser herramientas importantes en la educación formal, ya que permiten desarrollar capacidades como la investigación, la comprensión histórica, crear opiniones personales, articular ideas y desarrollar una escucha activa.

En el contexto de las aulas virtuales, donde el medio principal para llegar a los estudiantes se ha convertido en las imágenes proyectadas en pantallas, la alfabetización visual y la capacidad de observación activa es esencial para aprender: "en primer lugar, mirar no es suficiente. La persona que mira también debe ver. Y ver es una cuestión de conocimiento, entendimiento o experiencia. Ver supone interpretar la importancia de lo que se ve" (Eisner, 2004).

Los programas educativos en versión digital utilizan el Aprendizaje Basado en Objetos (ABO) para la exploración guiada de obras seleccionadas mediante el uso de los sentidos. Eilean Hooper - Greenhill explica cómo el uso de la metodología ABO no solo es importante por la información que nos brinda un objeto, sino porque además genera la curiosidad por nuevos conocimientos y experiencias:

Esta información es discutida, relacionada con conocimientos y experiencias previas y comparada con la percepción de otros. La síntesis de material exige más información y puede promover la investigación por parte del profesor, del alumno o de ambos a la vez. (1996, p. 232)

En este sentido, el programa "Nuestra Vestimenta" invita a los estudiantes a explorar la textura de las motas de algodón que tengan en casa y compararlas con la planta de algodón nativo que mostramos en el video (Fig.2) y que es utilizado para la elaboración de la cushma ${ }^{9}$ tradicional asháninka (prenda de vestir usada en la Amazonía peruana). De esta manera, a

\footnotetext{
${ }^{9}$ Prenda parecida a una túnica larga que llega hasta los pies. Es la vestimenta tradicional de los pueblos asháninka, amuesha-yánesha, matsiguenka y yine, de la Amazonia peruana.
} 
través del sentido del tacto y de la vista, los estudiantes pueden interpretar una obra detonando el interés y comprensión de temas específicos.

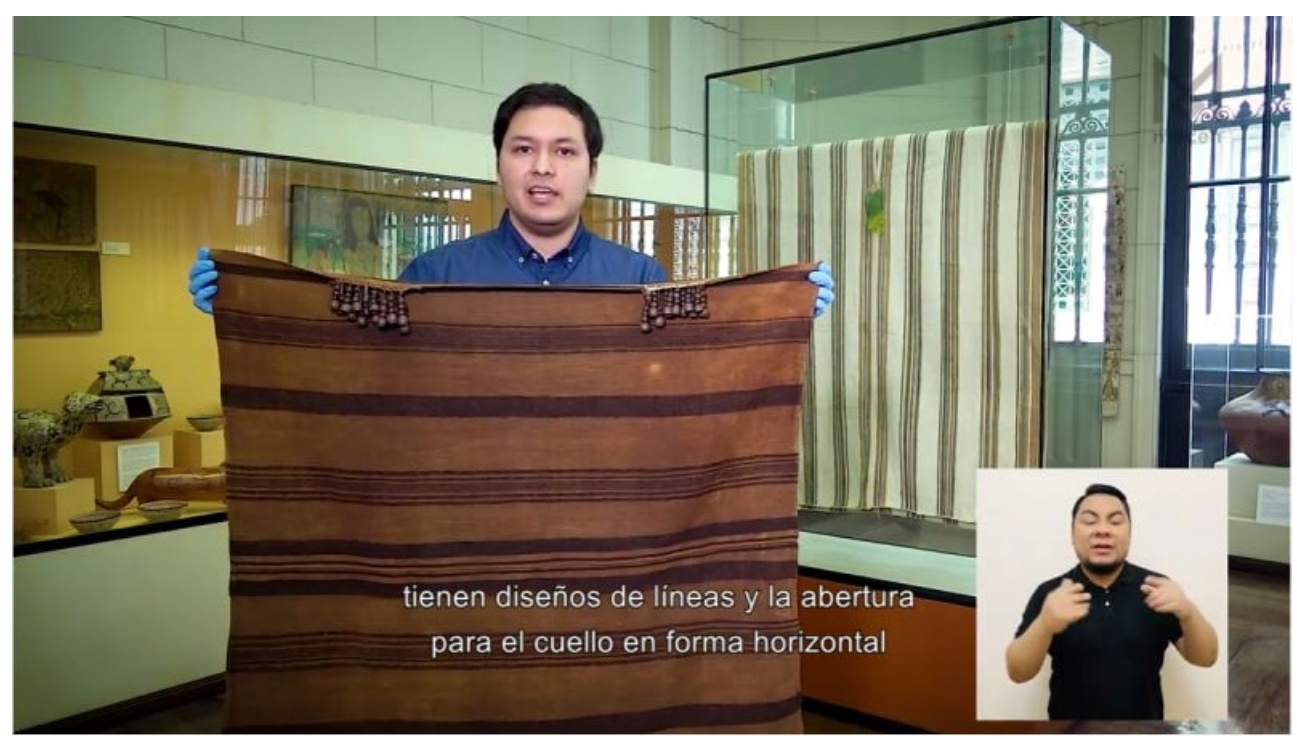

Fig. 2: Video interactivo Vestimenta tradicional asháninka

\subsubsection{Incentivando el diálogo y la interacción}

Las preguntas son herramientas esenciales en la mediación cultural, ya que permiten iniciar conversaciones, motivar la participación, promover nuevos descubrimientos y, sobre todo, invitar a los alumnos a formular más preguntas. En este sentido, los programas educativos toman como referencia los postulados de Howard Gardner (1985) quien propone desarrollar preguntas abiertas, partiendo de la observación, y valorando la experiencia y saberes previos de cada persona. Esto permitirá que el docente pueda ampliar conocimientos y reflexiones sobre temas transversales a las piezas que se muestran en los videos interactivos. Por ejemplo, plantear preguntas sobre las acuarelas del artista afroperuano Francisco "Pancho" Fierro podría generar conversaciones sobre las prendas de vestir actuales, los roles en la sociedad y las múltiples identidades.

Estas metodologías, ampliamente aplicadas por los mediadores en las salas del MUCEN, hoy constituyen herramientas puestas a disposición de los docentes peruanos. Estas pueden ser empleadas en la educación formal logrando que los estudiantes cumplan un papel protagónico en su proceso de aprendizaje y que las familias y otros agentes sociales descubran que la escuela y el museo son espacios próximos y complementarios para lograr aprendizajes significativos. 


\subsubsection{Aprendizaje desde las emociones}

Mikel Asensio y Elena Pol mencionan que "los museos no solamente se pueden basar en conocimientos intelectuales, sino que deberían aprovechar toda su carga emocional y actitudinal, y enlazar así con el componente más emocional del ser humano" (2008, p. 58). Desde el MUCEN asumimos la tarea de trabajar por crear espacios más amables para el aprendizaje, por lo que los programas educativos en versión digital, buscan que las emociones se generen incluso antes de iniciar el recorrido a través de propuestas de microactividades que motiven la curiosidad, el interés y la identificación de saberes previos.

Incluso el tratamiento de las temáticas, especialmente aquellas que puedan generar incomodidad, son abordadas desde una posición empática. Tal es el caso del programa educativo "Cosmovisiones" en el que se hace una aproximación a las diferentes concepciones sobre la muerte.

En la guía para docentes, se plantea una sección llamada "Incentivando el diálogo" que propone una serie de preguntas para que los estudiantes puedan identificar sus emociones y la de los demás, así como generar diálogos a partir de estas. Las preguntas hacen hincapié en el entendimiento de la identidad colectiva y personal que se ve reflejada en las obras de arte exploradas. Así, por ejemplo, para la pintura La Muerte de Sócrates (Fig. 3) se plantea la siguiente pregunta: ¿Qué emociones crees que están sintiendo los personajes del cuadro? ¿Qué te hace pensar eso?

De esta manera se busca no solo atender el carácter académico de las piezas de arte sino conectar con las historias que estas nos cuentan a través de sus personajes y validar las múltiples interpretaciones de los estudiantes.

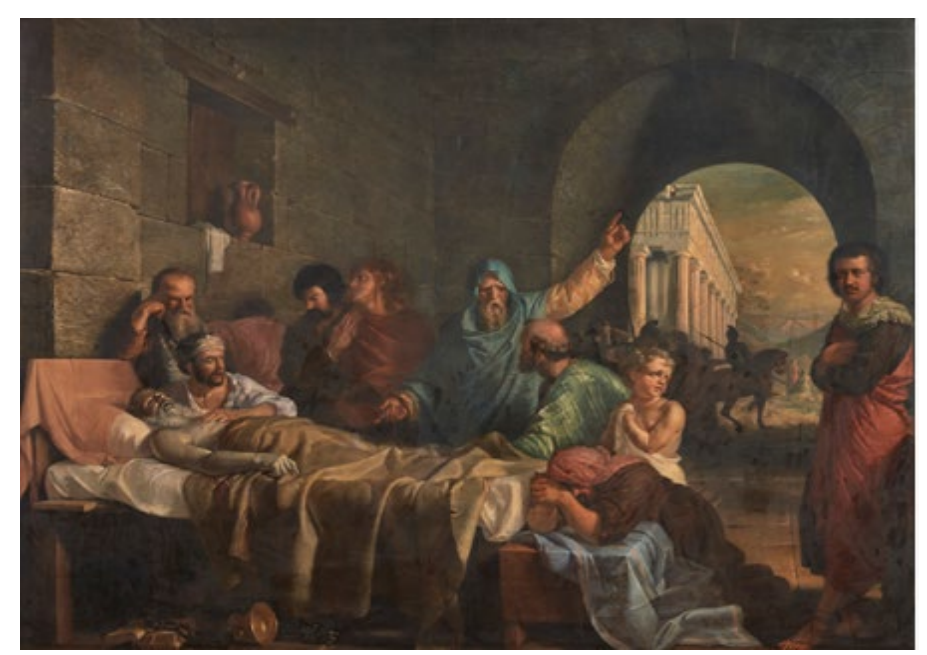

Fuente: Catálogo de Colecciones MUCEN

Fig. 3: La Muerte de Sócrates (1872) de Daniel Hernández 


\section{Resultados}

Los programas educativos en versión digital presentados son el resultado de una rápida respuesta del Museo Central ante el contexto de distanciamiento físico producto de la pandemia del COVID-19. Esta situación ha permitido ampliar las fronteras del museo fuera de sus paredes, a las aulas, escuelas y hogares de nuestro público escolar y docente a nivel nacional, manteniendo nuestra labor educativa y permitiendo el diálogo, mediación e intercambio de saberes desde lo virtual.

La inclusión de la pestaña "Aprende" en la web del MUCEN nos ha permitido centralizar los materiales desarrollados y facilitar la evaluación cuantitativa basada en la cantidad de personas que ingresan a revisar los recursos digitales. Así mismo, con el objetivo de poder contactarnos de manera personalizada con los docentes, se ha colocado un formulario previo a la descarga de la guía. Esto nos permitirá ofrecerles una sesión de asesoramiento en caso no hayan realizado aún el programa educativo y enviarles una encuesta para evaluar su experiencia cuando lo hayan implementado.

Además de la web, los videos interactivos de los programas educativos se encuentran disponibles en las cuentas de Facebook, Instagram y Youtube del MUCEN. En esta primera fase, se ha logrado evaluar el alcance de visitas a la página web a través de la herramienta Google Analytics, la cual reporta un total de 1470 personas que han accedido a la pestaña "Aprende" que alberga los programas educativos. Por otro lado, las métricas de Facebook y Youtube indican un total de 22284 visualizaciones de los videos interactivos hasta la fecha.

En una segunda etapa de evaluación buscaremos establecer vínculos a largo plazo con docentes que nos permita conocer a profundidad sus experiencias y percepciones en torno a la propuesta educativa digital del MUCEN. Para ello, se plantea utilizar herramientas como grupos focales con docentes para identificar sus hábitos, niveles de satisfacción y la relevancia de los recursos educativos desarrollados. Esta información será un insumo para plantear e implementar mejoras que se ajusten a las necesidades de los docentes, tales como cubrir la demanda de diversas áreas curriculares, mejorar nuestras metodologías, entre otras.

Finalmente, a través de estos programas hemos logrado posicionar al Museo Central como un agente dinamizador de las artes y de los procesos educativos a nivel nacional. Este reconocimiento se evidencia en la inclusión de diversos recursos educativos producidos por el MUCEN en el programa "Aprendo en Casa” del Ministerio de Educación ${ }^{10}$.

${ }^{10}$ Servicio multicanal de educación a distancia por televisión, radio e internet. 


\section{Rutas a seguir}

El principal reto que enfrentan los educadores de museos es profundizar en las metodologías de evaluación de los programas desarrollados, los cuales deben ser compatibles con el medio digital. Esto se debe a que muchas de las iniciativas educativas surgieron como respuesta al contexto adverso de la pandemia del COVID-19, para mantenerse cercanos a sus comunidades y no necesariamente contaron con el tiempo suficiente para la planificación y ejecución de herramientas de evaluación. Por ello, una de las rutas que seguiremos es establecer vínculos más cercanos con instituciones educativas que nos permitan realizar evaluaciones personalizadas y a profundidad sobre la aplicación de los programas educativos.

A partir del desarrollo de los programas educativos "Cosmovisiones" y "Nuestra Vestimenta", hemos identificado que la mediación digital es una metodología que puede ser aplicada de manera exitosa en el ámbito educativo, donde el docente es un agente clave del proceso. Por esta razón, hemos realizado cuatro talleres sobre metodologías pedagógicas beneficiando alrededor de 100 docentes de diversas zonas del Perú. Así mismo, nos encontramos organizando el encuentro virtual "Comunidad de Educadores: I Encuentro Museos + Escuela" que busca ser un espacio de intercambio de experiencias para dar a conocer los recursos educativos digitales que los museos peruanos ponen a disposición de las instituciones educativas. En esta misma línea, buscaremos desarrollar el programa "Docente aliado" que permita la creación de una red de docentes aliados del MUCEN para el intercambio de conocimientos, experiencias y propuestas metodológicas, así como fomentar el trabajo colaborativo y la co-creación.

Con el objetivo de seguir fortaleciendo nuestras relaciones con la escuela, se ha diseñado y convocado al primer concurso "El MUCEN como recurso educativo", dirigido a los docentes de todas las asignaturas. Este busca promover la creación de proyectos de aprendizaje de manera interdisciplinaria haciendo uso de las colecciones del museo. Los proyectos ganadores serán publicados para que puedan ser replicados en las escuelas a nivel nacional.

Finalmente, los resultados presentados muestran un panorama favorable para continuar diseñando y ejecutando programas educativos en versión digital. Por esta razón, en el año 2021, se presentará el nuevo programa "La otra cara de la moneda" que abordará temas vinculados a la educación financiera, a través de la exploración de una selección de monedas para analizar sus procesos de fabricación y las historias detrás de estas. Además, en el marco de las celebraciones por el Bicentenario de la Independencia del Perú, se desarrollarán dos programas educativos que inviten a valorar la diversidad cultural e imaginar una Nación en común. 


\section{Referencias}

ALDEROQUI, S. (1996).” Museo y escuela: Una sociedad posible (entre prólogo y artículo) en Alderoqui, S. Museos y escuelas: socios para educar. Buenos Aires, Paidós.

ASENSIO, M. y POL. E (2008). “Conversaciones sobre el aprendizaje informal en museos y el patrimonio" en Fernandez, H. Turismo, Patrimonio y Educación Los museos como laboratorios de conocimientos y emociones. Lanzarote: Escuela Universitaria de Turismo de Lanzarote; Cabildo de Lanzarote. Área de Educación y cultura.

EISNER, E. (2004). El arte y la creación de la mente: el papel de las artes visuales en la transformación de la conciencia. Barcelona: Editorial Paidós.

GARDNER, H. (1985). Frames of Mind: The Theory of Multiple Intelligences. New York: Basic Books.

HEIN, G (1998). "The Constructivist Museum" en Hein, G. Learning in the Museum. Londres: Routledge

HOOPER-GREENHILL, E. (1996). "Museum education” en Hooper-Greenhill, E. The Educational Role of the Museum. Londres: Routledge.

HOOPER-GREENHILL, E. (2007). "Museums: learning and culture" en Hooper-Greenhill, E. Museums and Education Purpose, Pedagogy, Performance. Londres: Routledge.

MUSEO CENTRAL. Informe de resultados "Encuesta para docentes: Recursos educativos del Museo Central. https://www.bcrp.gob.pe/docs/ProyeccionInstitucional/Museo/encuesta-docente-2020.pdf [Consulta: 26 de febrero de 2021]

MUSEO CENTRAL. Experiencia MUCEN 2018: Análisis de resultados y oportunidades. https://www.bcrp.gob.pe/docs/Proyeccion-Institucional/Museo/memoria-mucen2018.pdf

[Consulta: 26 de febrero de 2021]

PASTOR HOMS, M (2011) Pedagogía museística. Nuevas perspectivas y tendencias actuales. Barcelona: Editorial Planeta. [un autor]

SATAUS, N. y FALK, J. (2017) "The Role of Emotion in Informal Science Learning: Testing an Exploratory Model" en Mind, Brain, and Education, vol. 11, issue 2, p. 4553. 